\title{
PhySiOTHERAPY SERVICE PROVIDERS' VIEWS ON ISSUES OF ASSISTANTS: Are Physiotherapy Assistants Needed?
}

\begin{abstract}
This paper presents views of physiotherapy service providers in selected areas of the Western Cape Metropolitan Region, over the issue of physiotherapy assistants and whether they are needed. Data were collected through semi-structured interviews. A thematic analysis resulted in five pre-determined categories. While the participants agreed that assistants are needed,

WAZAKILI M, DIP. PT, BSc Hons PT'; MPOFU RMB, PhD, MSc Rehab, MCSP'

${ }^{1}$ Department of Physiotherapy, Faculty of Community and Health Sciences, University of the Western Cape. they had divergent views over issues of name and role of assistants. The lack of both standardised training for assistants and knowledge of delegation by professionals was highlighted. The training of assistants should be examined seriously if the shortage of physiotherapy services in general has to be alleviated, suggesting that the recommendations of the national qualification framework (NQF) should be applied to the training of physiotherapists together with the assistants.
\end{abstract}

\section{KEYWORDS: PHYSIOTHERAPY ASSISTANTS, CARER, CRWS, THEMATIC ANALYSIS, AND TRAINING.}

\section{INTRODUCTION}

In Africa, physiotherapy is not accessible to many people because of a shortage of professionals caused by the lack of training in some countries and inadequate numbers of qualified staff in others. Consequently, personnel other than physiotherapists perform services that are physiotherapy related. In this paper, the term physiotherapy service providers includes personnel who, with or in the absence of physiotherapists, perform some services that are normally done by physiotherapists and the managers of organisations which include physiotherapy services.

At international and national conferences, physiotherapists have expressed

\section{CORRESPONDENCE:}

Mpofu RMB

Senior Lecturer,

Department of Physiotherapy,

Faculty of Community and

Health Sciences,

University of the Western Cape,

P O Box X17

Bellville

7535

Tel: (021) 959-2542 (w)

Tel: (021) 671-4648 (h)

Fax: (021) 959-2804 (h)

Email: rmpofu@uwc.ac.za divergent views over the need for assistants, their name, role and training. Physiotherapy assistants' training is different from country to country, from province to province and even within a province. The difference between physiotherapy assistants and other groups of midlevel workers, like grassroots workers, support workers, community rehabilitation workers (CRWs), is not often clearly defined. These names may signify the different roles that assistants play in their different work settings. These roles more often include some physiotherapy skills.

Health and social policies are placing emphasis on the community as the arena for care, a concept that has implications for service providers, service recipients and informal carers. The perpetual shortage of physiotherapists and lack of physiotherapy assistants places the burden of care on informal carers (Kirk 1998).

Despite the many views in support of the role assistants play in physiotherapy services and in rehabilitation, a small survey of outpatients departments in the UK showed that some physiotherapists did not recognise the need for training assistants (Ellis et al 1998). Here are some of the arguments pointed out by Saunders (1997) and Lee (1997) against training and working with assistants:
- Basic physiotherapy skills can be passed on without the need for one to have formal training.

- Flooding physiotherapy services with assistants lowers the standards of practice. If and when these assistants are not supervised due to shortage of staff, they pass as physiotherapists.

- Governments cannot afford to fund or run both physiotherapy and assistants training schools, and therefore should stick to training at professional level only.

- All training should be at degree level since anything less is being devalued.

- Independent mushroom training of assistants ... has placed considerable difficulties on the system because of lack of a consistent approach to assistants' role and training.

Saunders (1996) argues that the role of physiotherapists is to make clinical diagnoses, plan and carry out treatment and be involved in teaching as well as carrying out research and administrative duties. Pressure of time makes this obligation difficult to meet (Parry and Vass 1997). Thus a trained assistant should be considered a valuable member of staff who should help to ease the burden of the chronic shortage of personpower. Furthermore, demographic and economic considerations constitute a pressing case in favour of assistants. 


\section{OBJECTIVES}

The research sought to get the views of physiotherapy service providers (PSPs) in selected areas in the Western Cape Metropolitan area, over issues surrounding the need for physiotherapy assistants.

\section{METHODOLOGY}

Four centres, a tertiary and a secondary hospital, two rehabilitation projects which provide a variety of health care services including physiotherapy and employed assistants, were selected from the Western Cape Metropolitan Region. Participants with hands-on experience with physiotherapy related services were selected as follows: 2 physiotherapy heads of sections, 3 physiotherapy clinicians, 2 physiotherapy assistants, 4 rehabilitation workers and 2 programme managers from community projects.

All the participants, but 4, were interviewed individually. The 4 participants at one centre requested to be interviewed in a group so as to support each other. Semi-structured interviews were conducted using open-ended questions and probes. The interviews were taperecorded. The data were transcribed and sent back to the participants for checking and corrections. The data were sorted into predetermined categories which were analysed on non-linear model (Marshal and Rossman, 1995). In order to respect participants' anonymity, only codes are used.

\section{RESULTS AND DISCUSSION}

Five main categories emerged from the analysis and each is discussed below by highlighting some of the quotations which were representative of what the participants said as well as referring to relevant literature.

\section{Need for physiotherapy assistants:}

The participants confirmed the shortage of physiotherapists, assistants or rehabilitation workers to meet the present demands for the service. They noted that there were areas of practice which specifically suited assistants rather than physiotherapists. The danger of assistants being treated as cheap labour is real. The following quotations illustrate some of the above interpretations.
Manager 'H': I definitely, recommend working with CRWs, because the need for rehabilitation in the community is great. The programme runs under the concept of primary health care, therefore, it needs CRWs. The financial constraints also make it necessary that we should work with this group of people who are cheaper to train and to maintain in terms of salary.

Manager 'I': Current statistics tell us that there is one OT to 23000 people. There are probably similar figures for physiotherapists. There is no way we could be able to train enough professionals to change the situation ... the trained people go to work abroad, while the country remains with chronic shortage of staff.

Rehab worker 'V': I think both are needed, because physiotherapy assistants live away from where the disabled are, while CRWs live within the community ...

Rehab workers ' $\mathbf{W}$ ' 'X' ' $Y$ ' ' $Z$ ': We are very short staffed, we cover large areas each.

The need for assistants was not questioned, since they are an important workforce for the whole health service delivery, especially in the communities. One participant suggested that it would be beneficial if assistants or rehabilitation workers were trained at -the same rate as the professionals, because the two groups are complementary to each other.

These views are different from those listed earlier against training and working with assistants. However, the need for assistants was expressed differently by the respondents depending on who they are and for what reason they thought the assistants were needed. The managers and assistants did not see their role as that of filling in where there are no therapists, but as a permanent one to fulfil certain needs in the provision of rehabilitation services. As Saunders (1996) argues, the role of physiotherapy involves many duties, some of which can be done by assistants acting as valuable members of staff who could help to ease the shortage of person-power.

2. Common name for physiotherapy assistants: On being asked whether there should be a common name for assistants, some respondents answered as follows:

Physiotherapist 'A': I have no problems with the name assistant, because that is what they are, they assist physiotherapists to do their work.

Assistant 'T': I have not thought about titles, titles do not make a person. However, ... one should be able to specialise in one's area of interest and not be grouped under one umbrella with everyone else.

Assistant 'U': I have no problems with the name assistants. It is personalities that matter The lack of clear-cut roles is what frustrates me. There is no way one can pretend to be a physiotherapist when you are not.

Physiotherapist 'E': There is a stigma attached to the name assistant. There is a need to move away from the stigmatising name to something like "health worker", which is broad.

Manager ' $I$ ': It is better to have rehabilitation workers who are given a variety of skills from occupational therapists, physiotherapists and social workers, because the demands in the community are higher and they require multidisciplinary approaches. Specific professional assistants would be limited in this kind of work. Besides the name assistants carries a stigma with it.

These quotations illustrate the divergent views the respondents have on the name "assistants". Respondents in tertiary and secondary hospital had different views from those working in community settings. By seeing the role of assistants as that of assisting physiotherapists to do their work, this could be interpreted as an attempt to deny the assistant the role of being an essential member of the rehabilitation team who could function in the absence of physiotherapists. The name is found limiting in that either by their own will or that of others, assistants may not perform to their full potential. The view that one should be able to specialise in one's own area of interest and not be grouped with every one else cannot be ignored. This could explain the dilemma faced by trainers who continue to train assistants for a specific need, be it at provincial, community or hospital level. 


\section{Role of physiotherapy assistonts}

In describing the role of assistants, some respondents implied that physiotherapy assistants do 'basic routine work'.

Physiotherapist 'B': Physiotherapists do the initial assessment and assistants take over from there. ... Assistants have developed their own skills of treating patients because of their many years of experience in this unit.

Physiotherapist 'C': Assistants are dissatisfied with their work. They would like to be physiotherapists, but they do not want to take responsibilities. This makes one hesitate to improve them. ... some of them are too intelligent for the basic work that they are doing, they actually should be doing more, but they can't, because they do not have the training. They need direct supervision, but the department does not have the staff for that kind of thing.

Assistant 'T': We were initially trained as "boys", to prepare a place for them (physiotherapists) to come and work and then to go after them to clean up their mess, very demeaning job indeed. The only contact with patients was in transfers and the unbelievable passive movements. I was tired of standing around, cleaning the place ... One day I assertively took a patient from a physiotherapist and asked them to watch me while I treated ... After this they were satisfied and they started giving us patients after initial evaluation by qualified staff. While you (researcher) sat there with the Physiotherapist, I was busy treating a post-operative patient on my own.

Rehab worker 'V': We see the clients first, because we live in the community with them, once we have established the problems and the needs of the clients, we consult with the physiotherapist or the occupational therapist for further input.

The literature also confirms that assistants' potential is under-utilised and that they are under-trained (Parry and Vass 1997), because of no clearly defined roles. Assistants work alongside physiotherapists in patient care and sometimes they are called upon to perform varying amounts of domestic and clerical work. These views concur with Loomis'
(1997) that physiotherapists could not clearly differentiate between their routine work and that of assistants. Some respondents assumed that assistants are not capable of performing challenging tasks, because of less training. CRWs with less periods of learning claimed that they were capable of doing more with the opportunities of continuing education.

\section{Training of physiotherapy assistants.}

Problems around issues of physiotherapy assistants were seen as a direct consequence of lack of national training. The physiotherapy assistants' training has been initiated by physiotherapists mainly, while physiotherapists and other rehabilitation workers have initiated that of CRWs. Some participants inferred that the assistants are poorly trained and they neither meet the expectations of the physiotherapists nor are they satisfied with their own performance. Inadequacies in both physiotherapy and assistants training were cited, implying that physiotherapists are trained to be self-sufficient, hence their difficulties working with assistants. The following comments illustrate some of these sentiments.

Physiotherapist 'D': The assistants received about a year's training. Their training was shallow, that is why all the assessments are done by physiotherapists ... It would be good if they were trained at a national level for unform standards of practice.

Physiotherapist 'E': Physiotherapy assistants are largely trained to take orders and not to think around the reasons why they do things, hence the lack of recognition for their work.

Assistant ' $U$ ': I received 18 months training in both theory and practice. The training was very shallow, I cannot do more, and therefore it is boring ... I would also like to feel responsible and useful to the patients.

Rehab worker ' $\mathbf{V}$ ': I received 3 months training ... and I have received ongoing training from occupational therapists and physiotherapists. The training was adequate, because it was supplemented by the ongoing training ... in future it would be better to start national training in the country so that if one leaves one place they can always work elsewhere in the country.

There is not a lot written about assistants' training in Africa in general, except Zimbabwe which runs a national 2 year rehabilitation technician course. Zimbabwe has registered success in terms of reaching more people for rehabilitation services, the benefits of which are seen through the wide coverage of their community-based rehabilitation programmes. However, shortages of professionals are still experienced, to the extent that many rehabilitation technicians work alone in large catchment areas without supervision from any rehabilitation personnel (O'Donnel 1991), despite the fact that the number of physiotherapists and occupational therapists is increasing. This confirms what Saunders (1997) mentions that the development of physiotherapy assistants should be matched with increased numbers of physiotherapists to ensure constant supervision.

In South Africa, it is said that in 1987 associations of occupational therapy, speech and hearing therapy and physiotherapy nominated a committee which was mandated to look into the training of CRWs. Their proposal for training was rejected by academic institutions, but they went ahead and trained them anyway and established a number of training centres around the country (Taukobong 1999). Taukobong further asserts that in 1987 the South African Society of Physiotherapy also discussed the training of CRWs, noting that some of them are currently employed in physiotherapy departments of certain hospitals, and are directly supervised by physiotherapists. Taukobong associates this seeming rejection or non-support of CRWs to ignorance of future health service development and to lack of clearly defined roles for this cadre.

\section{Delegation}

Most PSPs perceived delegation to assistants as a problem. The physiotherapy assistants felt frustrated by the type of tasks delegated to them. They attributed the complexity of the matter to several factors as illustrated by the following quotations: 
Physiotherapist 'E': Delegation is a problem because physiotherapists are not trained to delegate, nor are they prepared to work with assistants. A physiotherapist is trained to think as an all-encompassing person ... It is necessary for physiotherapists to learn some delegation skills. The demand for services in the community is very high and the only way to stretch the limited staffing situation is to delegate to assistants. Clearly defined job descriptions would ease the problems ... Our training isolates us, we do not learn to work as a team. Teamwork must be incorporated into training, and then it will filter through our work situation.

Physiotherapist ' $\mathrm{C}$ ': Yes, delegation is a problem, one would rather do all the work alone because then you are sure that your work is done.

Manager 'I': The problem is with professional training that does not prepare people to work in a team, but prepares them to protect the profession from unknown ills.

Assistant ' $T$ ': After a year we started to challenge the system. We were frustrated with the boring routine... the only contact we had with patients was transfers and the unbelievable passive movements.

Loomis et al (1997) say that a lack of specificity of roles is what leads to problems with delegating responsibility. Further, Saunders (1998) suggests that delegation can be organised to improve efficiency, but physiotherapists are not investing in delegation due to lack of recognition of the potential value of delegation. It may also be easier for physiotherapists to perform the tasks themselves rather than to build the skills in assistants. Saunders also argues that task analysis is necessary to enable decisions to be made on whether subtasks are knowledge-, rule- or skill-based and thus suitable for delegation.

\section{CONCLUSION AND RECOMMENDATIONS}

This paper presents the varying views of physiotherapists, physiotherapy assistants, rehabilitation workers and programme managers in selected areas of the Western Cape Metropolitan area. All respondents would like to see changes for the better, in terms of assistants' training and role. They mentioned the need to increase numbers of staff by training both physiotherapy assistants and other groups of midlevel workers depending on their function. This is in line with what Loomis (1997) said that an expanded role for support personnel is a viable means of increasing availability to physiotherapy services.

The assumption is that this paper has not only clarified issues, but that present and future policies regarding the training of physiotherapy assistants will be influenced positively. Tireless efforts must be made for physiotherapy services to reach the communities that need it most, but are disadvantaged by the present system whose operations are limited by shortage of staff.

An attempt to clarify the roles is through the national qualification framework (NQF) which was established to provide for registration of national standards and qualifications in South Africa. The NQF states that competency in any particular field will be achieved through capacity for continuing performance within specified ranges and contexts resulting from the integration of a number of outcomes. The recognition of competence in this sense is the award of a qualification. As learners meet the criteria which show they have achieved the outcomes for required unit standards, they accumulate credits towards a particular qualification. When they have the required number of credits they achieve the defined degree of competence in that area and receive a qualification (Academic Development Bureau 1998).

Physiotherapy educators should be challenged to consider the all-encompassing nature of the NQF, perhaps by allowing the assistants to acquire enough credits to become physiotherapists eventually. There is no doubt that continued undefined separate training retards the intentions of the South African Qualifications Authority and the job satisfaction of physiotherapy assistants.

\section{REFERENCES}

Ellis B, Ellis-Hill C, Connell N A D 1997 Role training and job satisfaction of physiotherapy assistants. Physiotherapy, 12: 608-616

Information service on higher education: Academic Development Bureau Newsletter, 8 (1) I-II

Kirk S 1998 Trends in community care and patient participation: implications for the roles of informal carers and community nurses in the UK. Journal of Advanced Nursing, 370-381

Lee L 1997 Does Africa need you? New Zealand Journal of Physiotherapy

Loomis J, Forward J, Hagler P, McMillan A, Swinamer J, Wessel J 1997 Current utilization of physiotherapy support personnel in Canada. Physiotherapy Canada, 284-291

Marshall C, Rossman B 1995 Designing qualitative research. Secong Edition, SAGE Publications, London and New Delhi

O'Donnel D 1993 The needs of rural rehabilitative clients and a physiotherapy programme which would meet these needs: Illustrated by an investigation of how rural mothers adjust to the disability of their cerebral palsied children. Department of Physiotherapy, University of Cape Town

Parry R, Vass C 1997 Training and assessment of physiotherapy assistants. Physiotherapy, 84 (1): $33-39$

Saunders L 1996 Managing delegation to physiotherapy assistants. Physiotherapy, 82 (4): $246-252$

Saunders L 1997 Issues involved in delegation to assistants. Physiotherapy, 83(3) 141-147

Saunders L 1998 Improving the practice of delegation in physiotherapy. Physiotherapy: 84 (5) 207-215

Taukobong N P 1999 The role of the community-based rehabilitation worker within the primary health care service of the Odi District. South African Journal of Physiotherapy, 55 (1) $19-22$ 